\title{
Measurement of a Topological Edge Invariant in a Microwave Network
}

\author{
Wenchao Hu, ${ }^{1}$ Jason C. Pillay, ${ }^{2}$ Kan Wu, ${ }^{3}$ Michael Pasek, ${ }^{2}$ Perry Ping Shum, ${ }^{1}$ and Y. D. Chong ${ }^{1,2, *}$ \\ ${ }^{1}$ Centre for Disruptive Photonic Technologies, Nanyang Technological University, \\ Singapore 637371, Singapore \\ ${ }^{2}$ Division of Physics and Applied Physics, School of Physical and Mathematical Sciences, \\ Nanyang Technological University, Singapore 637371, Singapore \\ ${ }^{3}$ State Key Laboratory of Advanced Optical Communication Systems and Networks, \\ Department of Electronic Engineering, Shanghai Jiao Tong University, Shanghai 200240, China
}

(Received 14 August 2014; published 6 February 2015)

\begin{abstract}
We report on the measurement of topological invariants in an electromagnetic topological insulator analog formed by a microwave network, consisting of the winding numbers of scattering matrix eigenvalues. The experiment can be regarded as a variant of a topological pump, with nonzero winding implying the existence of topological edge states. In microwave networks, unlike most other systems exhibiting topological insulator physics, the winding can be directly observed. The effects of loss on the experimental results, and on the topological edge states, are discussed.
\end{abstract}

DOI: 10.1103/PhysRevX.5.011012

\section{INTRODUCTION}

Topological insulators are phases of matter that are "topologically distinct" from conventional insulators, meaning that their electronic band structures cannot be deformed into conventional band structures without closing band gaps. They have many striking physical properties, the most notable being the existence of "topological edge states," which, in two-dimensional topological insulators, have the unique property of being protected against backscattering from impurities. Topologically nontrivial band structures were originally discovered in condensed matter systems, first in the quantum Hall $(\mathrm{QH})$ effect [1] and later in materials with strong spin-orbit coupling [2]. Recently, the concept has been extended to photonics, with coherent classical electromagnetic fields taking the place of electron wave functions [3-14]. Such "topological photonics" devices have been realized with microwave-scale magnetic photonic crystals [3-6] and meta-atom structures [7], and, at optical and infrared frequencies, with waveguide lattices [8] and resonator lattices $[9,10]$. There have also been theoretical proposals based on modulated photonic crystal resonances [11], circuit QED systems [12,13], metamaterial photonic crystals [14], etc. The key feature of these devices is the existence of topologically protected electromagnetic edge states, which may have technological promise for waveguides that are robust against disorder.

\footnotetext{
*yidong@ntu.edu.sg
}

Published by the American Physical Society under the terms of the Creative Commons Attribution 3.0 License. Further distribution of this work must maintain attribution to the author(s) and the published article's title, journal citation, and DOI.
Subject Areas: Condensed Matter Physics,

Optics, Topological Insulators
Topological photonics may also prove useful for studying aspects of topological insulator physics that are difficult or impossible to probe in the condensed-matter context, such as the effects of nonlinearity [15].

In condensed-matter systems, the most important physical consequence of topologically nontrivial band structures is that they cause certain macroscopic transport properties to be precisely quantized. Most famously, in the integer $\mathrm{QH}$ effect, the Hall conductance is quantized to integer multiples of the inverse von Klitzing constant to nearly one part in $10^{9}$ [16]. An influential explanation for this was supplied by Thouless et al. [17], who showed using linear response theory that the $\mathrm{QH}$ conductance is tied to the Chern numbers of the bands, which are "topological invariants" restricted to integer values. In photonics, however, there is no direct analog of the Hall conductance or similar linear response-based quantity, due to the absence of a fermionic ground state $[3,4]$; this is why edge propagation measurements have served almost exclusively as the signature for topologically nontrivial photonic band structures.

In this paper, we present experimental measurements of a topological edge invariant in a microwave network. The invariant consists of the integer winding numbers of scattering matrix ( $S$ matrix) eigenvalues, measured at the edges of the sample. The experiment is a variant of the "topological pump," a thought experiment invented by Laughlin to explain the QH effect in topological terms-in a way that does not rely on linear response $[18,19]$. In Laughlin's original setup, the Hall conductance is described in terms of the adiabatic "pumping" of single-electron wave functions across the surface of a cylinder; due to the cylindrical geometry, the pumping can be expressed as the effect of a gauge transformation, whereupon the 
robustness of the $\mathrm{QH}$ effect follows as a result of the exactness of gauge invariance. The topological pump can also be formulated in terms of scattering processes [20-22], which is a particularly natural approach for describing electromagnetic systems [23]. As described below, the present experiment is based on this scattering formulation. It is remarkable that, even though the topological pump is typically regarded as a "thought experiment," it can actually be implemented in electromagnetic systems. Recently, topological pumps have been demonstrated in 1D quasicrystalline arrays of optical waveguides [24,25], and a related scheme based on a ring of coupled optical resonators has been proposed by Hafezi [26]. Compared to these previous works, our microwave experiment is noteworthy in that the measurements are not limited to intensities, but include full phase information, since the complex $S$ matrix can be determined with a microwave network analyzer. This allows the winding process to be directly observed. The difference between topologically trivial and nontrivial behaviors is also clearly observable in this system. The setup also provides a platform for studying the topological properties of "Floquet" or "quasienergy" band structures, a topic of current theoretical interest [27-33].

The remainder of this paper is organized as follows: Sec. II reviews the theory of network band structures and topological pumps, Sec. III describes the experimental setup and results, and Sec. IV discusses the implications of the experiment, including how we can understand the effects of losses on the topological edge invariant and the network band structure.

\section{BAND STRUCTURES AND TOPOLOGICAL PUMPS IN NETWORKS}

\section{A. Network band structures}

Our realization of an electromagnetic topological insulator is a network implemented with microwave components. Such a system is best understood using the framework of a "network model" [34], which differs in several respects from the tight-binding Hamiltonian models familiar to most readers. This section describes our network model and its topological properties. A more detailed discussion may be found in Ref. [23].

Network models, which originated in the $\mathrm{QH}$ literature as a convenient way to study disordered QH systems [34] and other types of topological insulators [35,36], describe directed networks within which waves can propagate. We consider the specific periodic (disorder-free) network shown in Fig. 1(a), which consists of unit cells arranged in a 2D square lattice, each cell containing two nodes and four directed links. A "solution" to the network is a set of wave amplitudes (complex scalars) defined along the links, such that (i) traversing each link incurs a phase delay $\phi$ (which we take to be the same for all links) and (ii) the amplitudes (a)

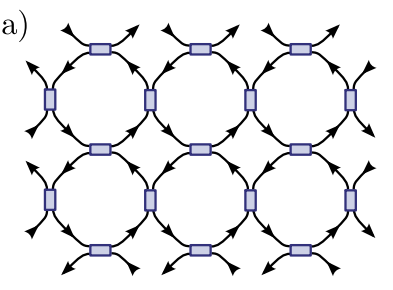

(b)

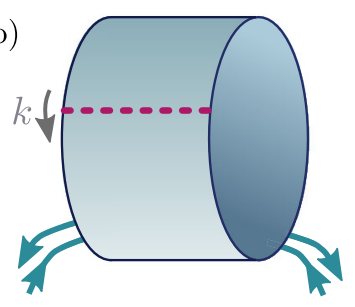

(c)

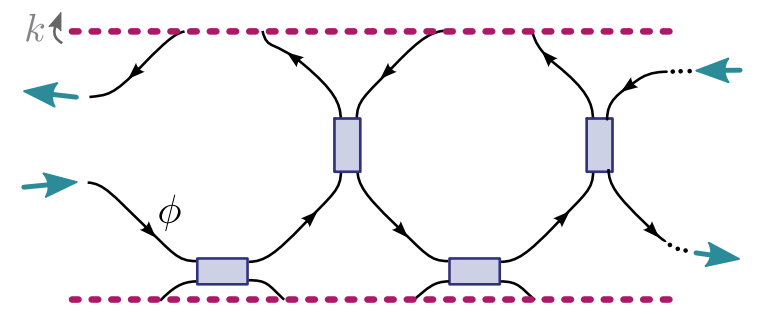

FIG. 1. (a) Schematic of a periodic 2D directed network. Wave amplitudes undergo phase delay $\phi$ along each directed link (black arrows) and couple through a $2 \times 2$ unitary matrix at each node (blue boxes). (b) In a "topological pump" setup, a 2D system is rolled into a cylinder, twisted boundary conditions with tunable twist angle $k$ are applied, and a transport measurement is performed in the axial direction (cyan arrows). (c) Topological pump setup for a network model, with a cylinder comprising one unit cell in the azimuthal direction and two cells in the axial direction.

entering and leaving each node are related by a fixed $2 \times 2$ coupling matrix. Let the complex vector $|\psi\rangle$ be the collective set of wave amplitudes exiting the links and $\left|\psi^{\prime}\right\rangle=e^{-i \phi}|\psi\rangle$ be the amplitudes entering the links. The individual node-coupling matrices can be composed into a matrix $U$ that relates the amplitudes entering and leaving the nodes. These amplitudes must be equal to $|\psi\rangle$ and $\left|\psi^{\prime}\right\rangle$, respectively, so

$$
U|\psi\rangle=e^{-i \phi}|\psi\rangle .
$$

For an infinite periodic network, we look for solutions obeying Bloch's theorem. Then, Eq. (1) reduces to [23,37]

$$
U_{k}\left|\psi_{n k}\right\rangle=e^{-i \phi_{n}(k)}\left|\psi_{n k}\right\rangle,
$$

where $n$ is the band index, $k$ is the quasimomentum, and $\phi_{n}(k)$ is the band quasienergy. In the absence of gain or loss in the network, $U_{k}$ is unitary and $\phi_{n}(k)$ is real. But unlike a usual band energy, it is an angle variable.

Interestingly, Eq. (2) has the same form as the evolution equation for a lattice with a time-periodic Hamiltonian, where $U_{k}$ is the evolution operator over one period and $\left|\psi_{n k}\right\rangle$ is a Floquet state of the driven lattice. Recently, several groups have proposed a new class of topological insulators called "Floquet topological insulators," where the topological behavior arises from periodic drives (such as oscillating electric fields). It has been shown that a driven system can have a topologically nontrivial quasienergy 
band structure, even if the undriven energy band structure is topologically trivial [27-33].

The network model framework is also useful for describing a type of photonic device proposed and realized by Hafezi et al., consisting of a lattice of ring resonators joined by auxiliary waveguides $[9,10]$. By engineering incommensurate modulations into the auxiliary waveguides, Hafezi et al. induced an effective tight-binding "vector potential" corresponding to a uniform magnetic field. The system thus maps onto a QH system and exhibits electromagnetic analogs of QH edge states [10]. Surprisingly, however, even if the auxiliary waveguides are symmetrical and commensurate, corresponding to zero "magnetic flux," full-wave simulations show that robust one-way edge states can still exist [38]. This is explained by moving beyond the tight-binding description and formulating a network model where the arms of the ring resonators are links $[23,37,38]$. That network model is equivalent to the one considered in this paper.

In most respects, quasienergy band structures have the same behaviors as energy band structures. If quasienergy bands have nonzero Chern numbers (as calculated from $\left|\psi_{n k}\right\rangle$ in the usual way [17]), topological edge states will exist; this is the case demonstrated in a recent photonic realization of a Floquet topological insulator [8]. However, a quasienergy band structure can also be topologically nontrivial even if all Chern numbers are zero [30]. This case, which we call the "anomalous Floquet insulator," is possible because $\phi_{n}(k)$ is an angle variable; for example, every band can receive +1 to its Chern number from the band above and -1 from the band below, resulting in zero net Chern number even though all band gaps are topologically nontrivial. For driven systems, Rudner et al. have proposed an alternative topological bulk invariant that can characterize this situation [33]. The topological nontriviality of an anomalous Floquet insulator can also be verified from the presence of topological edge states, and from its behavior under topological pumping (as described in the next section) [23].

For the periodic network of Fig. 1, the quasienergy band structure can be obtained analytically [37]. It turns out that the band topology depends on a single parameter $\theta \in[0, \pi / 2]$, which describes the coupling strength at the network nodes. The band gaps close at $\theta=\pi / 4$. For $\theta<\pi / 4$, the system is a topologically trivial conventional insulator; when $\theta>\pi / 4$, the system is an anomalous Floquet insulator [23]. In the experiment, this allows us to switch easily between topologically trivial and nontrivial behaviors.

\section{B. Topological pumps}

A topological pump is an experiment that is specially designed to reveal the topological properties of a 2D lattice. As shown schematically in Fig. 1(b), it consists of rolling a 2D lattice into a cylinder, inducing a phase "twist" $k$ in the azimuthal boundary conditions, and performing a transport measurement in the axial direction (i.e., at the edges of the cylinder). In the original Laughlin thought experiment [18], the twist is implemented by adiabatically threading magnetic flux through the cylinder, which produces an Aharonov-Bohm phase shift while also transporting electron wave functions in the axial direction. Threading one magnetic flux quantum induces a $2 \pi$ phase shift; by gauge invariance, the number of transported electrons must be an integer. From this, the quantization of the Hall conductance can be derived [18].

An alternative formulation of the topological pump, based on wave scattering, has been developed by Brouwer and co-workers [20,21]. Here, one imagines taking a similar cylinder and scattering electron waves (or electromagnetic waves, as the case may be) off one edge, at an energy (or frequency) that lies in a bulk band gap. As the twist angle $k$ advances by $2 \pi$, the reflection matrix $r$ is measured. For a sufficiently long cylinder and/or a sufficiently large band gap, transmission to the opposite edge is negligible and $r$ is unitary; its eigenvalues lie on the unit circle, and their trajectories during a pumping cycle can be topologically characterized by a winding number. A nonzero winding number corresponds to a topologically nontrivial sample. This is tied to the existence of topological edge states. In a topologically nontrivial system, edge states must occur at certain values of $k$ during the pumping cycle [19]. According to scattering theory, each such occurrence induces a $\pi$ scattering phase shift [21]. Such a phase shift can be guaranteed only if the eigenvalue trajectories have nonzero winding. This behavior is "topologically protected," since weak perturbations deform the trajectories of the reflection eigenvalues without altering the winding number, and the only way to remove the winding is to close the band gap.

Our experiment implements a similar scheme, in the context of a microwave network, as shown in Fig. 1(c). The tunable twist $k$ is implemented by connecting the links (microwave cables) at each end of the "azimuthal axis" to phase shifters. We measure the full scattering parameters along the axis (i.e., the complex reflection and transmission coefficients for microwave signals injected into the edges of the cylinder), thus obtaining the $S$ matrix, which is $2 \times 2$ for this network geometry. In the ideal situation, where the network is completely lossless, $S$ is unitary, and its eigenvalues $\sigma_{ \pm}$lie on the unit circle. If the quasienergy $\phi$ lies in a sufficiently large band gap, then the transmission goes to zero and $\sigma_{ \pm}$reduce to the reflection coefficients from each edge (this condition can indeed be met, as discussed in Sec. IV). The winding numbers of $\sigma_{ \pm}$, as the twist angle $k$ is tuned through $2 \pi$, are the desired integer invariants [20-23].

Several other schemes to directly measure topological invariants have recently appeared in the literature. Most prominently, a topological pump has been realized 
experimentally using a 1D quasicrystalline optical waveguide array, which can be mapped formally to a $2 \mathrm{D} \mathrm{QH}$ system $[24,25]$. A scheme to implement a topological pump in a 2D system has recently been proposed by Hafezi [26]. Here, coupled resonators are arranged in an annulus, with a tunable phase shift along one column of the annulus; such a configuration is similar to the Laughlin thought experiment [18] and to the present experiment. A topological invariant is then inferred from the transport of resonance peaks in the transmission spectrum along the edge. This proposal has yet to be realized.

Topological pumps, such as the present experiment and the related schemes described in the preceding paragraph, are probes of topological edge invariants. There have also been theoretical proposals to directly measure topological bulk invariants-specifically, Chern numbers-in photonic systems $[39,40]$. Typically, a bulk invariant is significantly more challenging to measure than an edge invariant, as it requires detailed information about the wave functions in the bulk, whereas the latter depends only on the response of the system at a single frequency or quasienergy [21]. Furthermore, as noted above, edge invariants can detect topologically nontrivial behaviors in cases where the band topology cannot be fully characterized by Chern numbers [23]. (A recent experiment has measured a topological bulk invariant in a specific 1D system [41].)

\section{EXPERIMENTAL SETUP AND RESULTS}

Our experimental setup is shown in Fig. 2. The network is divided into identical subunits, each consisting of four cables, two phase shifters, and two couplers. By comparison with Fig. 1(c), each subunit is equivalent to a "cylinder" in the topological pump setup that is one cell wide and one cell long. Connecting the subunits in series forms a longer cylinder. Figure 2 shows a two-cell configuration. The

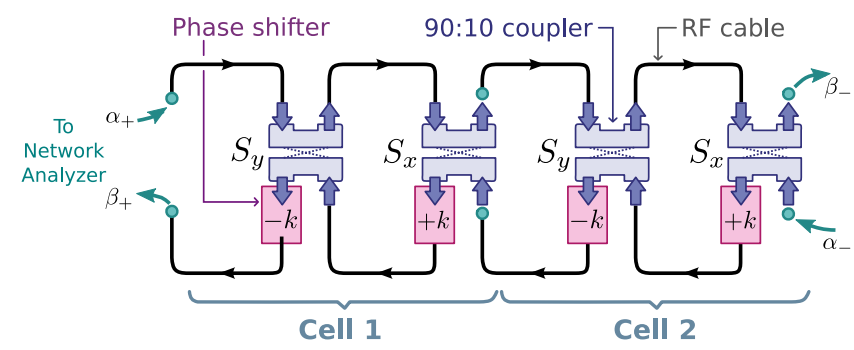

FIG. 2. Experimental setup. Each of the identical units, labeled "Cell 1" and "Cell 2," corresponds to one cell in the topological pump geometry of Fig. 1. A pair of phase shifters in each cell (pink boxes), with shifts of $+k$ and $-k$, respectively, implement the twisted boundary condition. The couplers (blue rods) are depicted in the strong-coupling configuration. The weakcoupling configuration is achieved by swapping each coupler's outputs. The overall input and output amplitudes are $\alpha_{ \pm}$and $\beta_{ \pm}$. Their scattering parameters are measured with a network analyzer. cables are standard low-loss coaxial RF cables of $\sim 15 \mathrm{~cm}$ length, and the couplers are four-port single-directional couplers, with isolators built into each port and coupling ratios of approximately 90:10 (DTO-2.5/5-10, Shanghai Huaxiang Computer Ltd.). The two phase shifters in each cell (TKE-90-6SA, Shanghai Huaxiang Computer Ltd.) are independently tunable and are set to produce $+k$ and $-k$ phase shifts, respectively. The operating frequency is chosen to be $5 \mathrm{GHz}$, low enough to reduce losses in the various components while high enough to allow phase shifts in the full range $k \in[0,2 \pi]$. There is one input port and one output port at each end of the network, connected to a vector network analyzer (Anritsu 37396C). From this, we measure the $S$ matrix, defined as

$$
S\left[\begin{array}{l}
\alpha_{+} \\
\alpha_{-}
\end{array}\right]=\left[\begin{array}{l}
\beta_{+} \\
\beta_{-}
\end{array}\right]
$$

where $\left\{\alpha_{ \pm}, \beta_{ \pm}\right\}$are the input and output wave amplitudes at the two edges, as labeled in Fig. 2.

Since the couplers have fixed coupling ratios, we are unable to continuously vary the network's coupling strength parameter, on which the band structure topology depends. However, by swapping the order of each coupler's output ports, we can switch between the two cases of a topologically trivial and nontrivial band structure. As described in Sec. II A and Ref. [37], the strength of each coupler is described by a parameter $\theta \in[0, \pi / 2]$, where $\theta=0$ corresponds to zero coupling between adjacent unit cells and $\pi / 2$ is complete coupling. The band structure is topologically trivial for $\theta<\pi / 4$, and nontrivial for $\theta>\pi / 4$, independent of all other coupling matrix parameters. Our 90:10 couplers thus allow for either $\theta \approx$ $\tan ^{-1}(3) \approx 0.40 \pi$ (topologically nontrivial; this is the configuration shown in Fig. 2) or $\theta \approx \tan ^{-1}(1 / 3) \approx 0.10 \pi$ (topologically trivial). At the $5 \mathrm{GHz}$ operating frequency, each cable has phase delay $\approx 0.2 \pi$, which lies in a band gap of the quasienergy band structure for both the strong- and the weak-coupling cases. The loss in each cable is $\approx 0.4 \mathrm{~dB}$.

The measured $S$ matrix eigenvalues are shown in Fig. 3. The eigenvalues do not lie on the unit circle, due to losses in the network that make the $S$ matrix subunitary. Nonetheless, the eigenvalue trajectories exhibit winding behaviors very similar to the lossless case. For the one-cell system under strong coupling, the two $S$ matrix eigenvalues, $\sigma_{ \pm}$, move along distinct closed trajectories as $k$ is increased through $2 \pi$, as shown in Fig. 3(a). Each individual trajectory does not encircle the origin (i.e., the winding number is zero), even though the network band structure is in the topologically nontrivial regime. This is because topological protection requires the opposite edges in a finite system to be well separated, so as to have negligible overlap between the counterpropagating edge states on each edge. When the separation is increased by connecting two cells in series, the trajectories coalesce into 


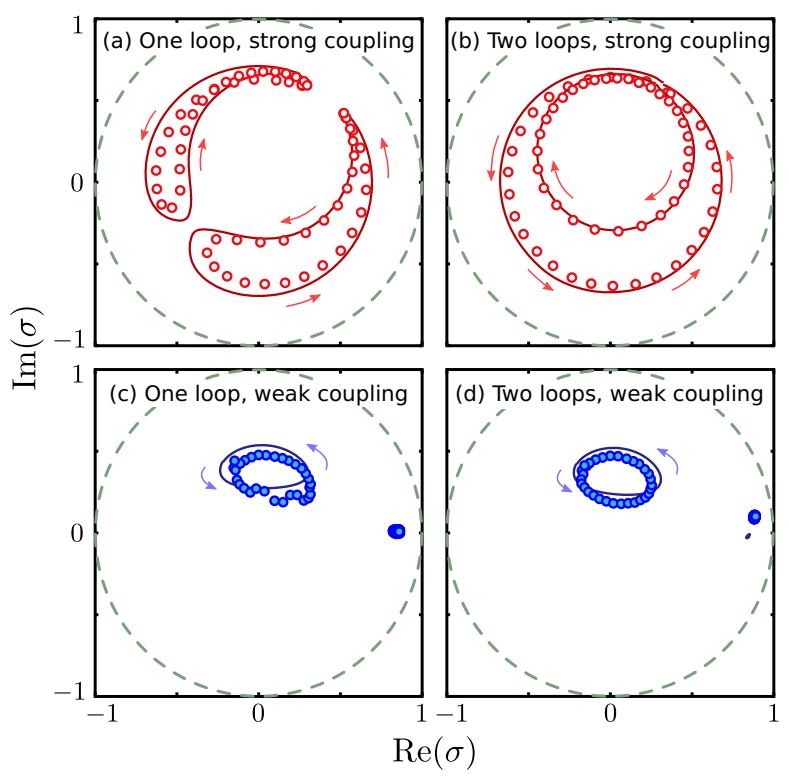

FIG. 3. Scattering matrix eigenvalues measured across one cell (left) and across two cells in series (right). Arrows indicate the direction of motion with increasing $k$. Circles show experimental data and solid curves show theoretical calculations using the scattering parameters measured for each network component individually (including losses). Nonzero winding numbers can be observed in the strong-coupling two-cell case. The unit circle is indicated by dashed curves.

a pair of loops with winding numbers \pm 1 , as shown in Fig. 3(b). By contrast, in the weak coupling regime of Figs. 3(c) and 3(d), the eigenvalues move along separate trajectories without encircling the origin.

The winding numbers can also be visualized by plotting $\arg \left[\sigma_{ \pm}\right]$against $k$, as in Fig. 4. It is also worth noting that, in the topologically nontrivial regime, the two $S$ matrix eigenvalues wind in opposite directions. This corresponds

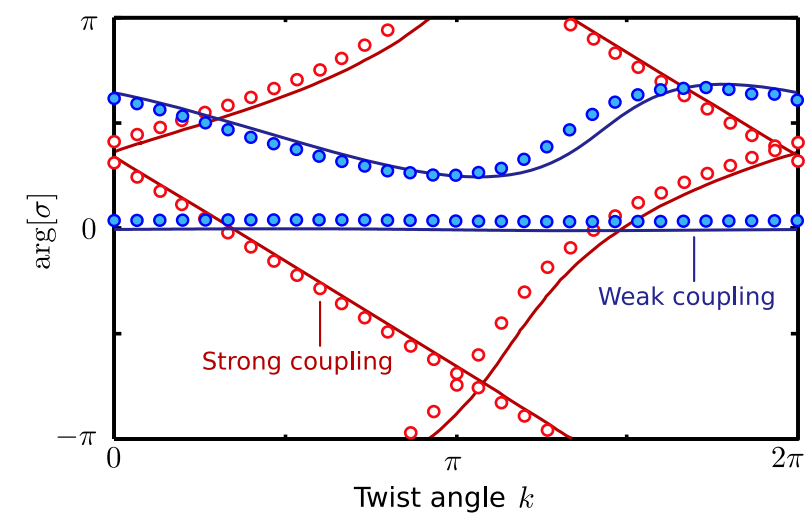

FIG. 4. Arguments of the complex $S$ matrix eigenvalues for the two-cell network, as the phase shift $k$ is tuned through $2 \pi$. Empty circles show the strong coupling measurement data, filled circles show the weak coupling data, and solid curves show theoretical calculations. For strong coupling, the two eigenvalues have winding numbers \pm 1 , corresponding to the bulk band structure being topologically nontrivial. to the fact that the topological edge states on opposite edges have opposite group velocities. In terms of the projected band structure, one branch of the edge state has a dispersion curve that crosses the probed value of $\phi$ from above and the other from below; hence, the induced scattering phase shifts have opposite signs.

\section{DISCUSSION}

Our experiment deviates from an ideal topological pump in several respects. First, as previously mentioned, in the ideal topological pump, the edges are separated by a large number of unit cells, so that there is a true "bulk," whereas in our experiment, there are only two unit cells. However, this does not make a significant difference to the physical interpretation, as the relevant phenomenon-the emergence of a nonzero winding number-is observed already when going from the one-cell to the two-cell case, shown in Figs. 3(a) and 3(b). This arises from the fact that the system is deep in either the topologically trivial or nontrivial phases, based on our choices of the coupling strength $\theta$; in the nontrivial case, each edge state is strongly confined to one unit cell, with negligible amplitude on the next unit cell. Indeed, calculations based on realistic parameters show no significant changes in the trajectory of the $S$ matrix eigenvalues as the number of cells is increased from 2 to 100 [42].

When the edges are well separated, the $S$ matrix reduces to a pair of reflection coefficients $r_{ \pm} \equiv \beta_{ \pm} / \alpha_{ \pm}$, where $\left\{\alpha_{ \pm}, \beta_{ \pm}\right\}$are the input or output wave amplitudes labeled in Fig. 2. This is observed in the small transmission coefficients in the two-unit cell systems: $|t|<0.06$ in the strong-coupling system and $|t|<0.04$ in the weakcoupling case. Simulations reveal that the edge states have penetration depths on the order of a unit cell [42].

Another difference between our experiment and the ideal topological pump is the presence of loss in all of the network components: the cables, phase shifters, and couplers. As a result, the eigenvalues of the $S$ matrix are not strictly constrained to the unit circle, as seen in Fig. 3. Nonetheless, we argue that these eigenvalue trajectories can be meaningfully linked to the existence or nonexistence of topological edge states, based on the close relationship between the edge scattering parameters measured in the experiment and the projected band structure of the network.

In the experiment, $r_{ \pm}$is determined based on two parameters $\phi$ and $k$, with all other parameters (such as the coupling matrices) fixed; varying $k$ then gives results like those shown in Figs. 3 and 4. Alternatively, we could either (i) determine $\phi$ given $r_{ \pm}$and $k$ (by finding the eigenvalues of the scattering matrix for one period of an infinite strip [23]) or (ii) determine $k$ given $r_{ \pm}$and $\phi$ (by finding the eigenvalues of the transfer matrix for one period of the strip). Both procedures yield the projected band structure, with $r_{ \pm}$ interpreted not as reflection coefficients but as boundary conditions that are applied along the edge of the strip, specifying the phase shifts on the edge links. In the absence 
of losses, if the scattering experiment yields a nonzero winding number for $\phi$ in a bulk band gap, then it can be seen that the projected band structure exhibits topological edge states [23].

Now, consider a lossy network. The losses can be described, without loss of generality, by making the coupling matrices subunitary, as well as by setting $\left|r_{ \pm}\right|<1$ (lossy boundary conditions). The two procedures described in the preceding paragraph, (i) and (ii), lead to two distinct types of complex projected band structure. We focus on procedure (ii), which takes $r_{ \pm}$and a real $\phi$ and yields complex wave numbers $k$, whose imaginary parts are the attenuation constants of the propagating modes. One such complex band structure is shown in Fig. 5, generated using representative lossy $r_{ \pm}$and coupling matrices (a fivecell-wide strip is used for clarity). The band structure contains clearly defined edge states whose dispersion curves have real parts nearly indistinguishable from the lossless network's topological edge states. Apart from these, there also exist propagating bulk states. Even in the range of $\phi$ corresponding to the lossless network's bulk band gap, there are bulk states with $\operatorname{Re}[k] \neq 0$, which are continuable to purely evanescent $(\operatorname{Re}[k]=0)$ states of the lossless system. However, these bulk states have significantly larger attenuation $|\operatorname{Im}(k)|$ compared to the edge states, which can be attributed to the fact that they experience losses over a wider area. If we wind $\arg \left[r_{ \pm}\right]$ through $2 \pi$, with fixed $\left|r_{ \pm}\right|<1$ (corresponding to a subunitary trajectory in the complex plane), the part of the complex projected band structure corresponding to the
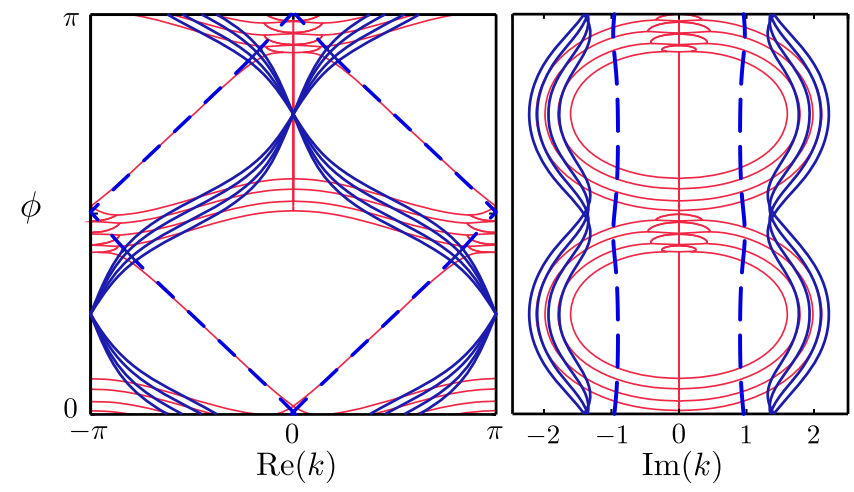

FIG. 5. Complex projected band structure of a hypothetical lossy network (blue lines), consisting of an infinite strip 5 unit cells wide. Each link has real tunable phase delay $\phi$, each coupler has loss $e^{-\gamma}$ in each output port where $\gamma=0.25$, and each edge of the strip obeys lossy boundary conditions $r_{ \pm}=\exp (-0.2 \pi)$, chosen to be roughly the same as in the experiment. The coupling strength is $\theta=0.4 \pi$, approximately equal to the "strong coupling" (topologically nontrivial) configuration in the experiment. The band structure for the lossless network $\left(\gamma=0\right.$ and $\left.\left|r_{ \pm}\right|=1\right)$ is plotted for comparison (red lines). The lossy network exhibits edge states whose dispersion relations are almost identical to the lossless network's topological edge states, and have lower loss than all other states (blue dashes). bulk states remains nearly unchanged, in both its real and imaginary parts. For the edge states, the real part of the dispersion curve winds with $\arg \left[r_{ \pm}\right]$, whereas the imaginary part remains nearly unchanged. Hence, winding $\arg \left[r_{ \pm}\right]$has the effect of "pumping" a branch of lossy edge states across one quasienergy period.

In summary, we observe a topological edge invariant, based on topological pumping, in a classical microwave network. Since networks of this sort are rather simple to set up, this may be a convenient method for studying the physics of topological band structures. For instance, we have discussed how the quasienergy band structures that arise in networks can have the same properties as the band structures of Floquet topological insulators [27-32]. In the square-lattice network chosen for the present experiment, the topologically nontrivial phase is an anomalous Floquet insulator, which possesses topological edge states despite all bands having zero Chern number [29]. In other network geometries, such as hexagonal lattices, different parameter choices give rise to either anomalous Floquet insulator phases, Chern insulator phases (in which the Chern numbers are nonzero), or conventional insulator phases [23]. If couplers with tunable coupling strengths are implemented, the transitions between these various phases could be directly observed. In future work, it would be desirable to reduce the losses in the network components, though it is encouraging that a nonzero winding is already clearly observable even at the current loss levels. Based on our above arguments, the edge states are physically meaningful despite the presence of loss; although both edge states and bulk states undergo attenuation, the two types of state are clearly distinguishable in the complex projected band structure. Since the edge states receive significantly less attenuation, they are the dominant mode of transmission along the edge.

\section{ACKNOWLEDGMENTS}

We are grateful to M. Hafezi, M. Rechtsman, B. Zhang, C. Soci, and N. Zheludev for helpful discussions. This research was supported by the Singapore National Research Foundation under Grant No. NRFF2012-02, and by the Singapore MOE Academic Research Fund Tier 3 Grant No. MOE2011-T3-1-005.

[1] M. Stone, Quantum Hall Effect (World Scientific, Singapore, 1992).

[2] J. E. Moore, The Birth of Topological Insulators, Nature (London) 464, 194 (2010).

[3] F. D. M. Haldane and S. Raghu, Possible Realization of Directional Optical Waveguides in Photonic Crystals with Broken Time-Reversal Symmetry, Phys. Rev. Lett. 100, 013904 (2008).

[4] S. Raghu and F. D. M. Haldane, Analogs of Quantum-HallEffect Edge States in Photonic Crystals, Phys. Rev. A 78, 033834 (2008). 
[5] Z. Wang, Y. D. Chong, J. D. Joannopoulos, and M. Soljačić, Reflection-Free One-Way Edge Modes in a Gyromagnetic Photonic Crystal, Phys. Rev. Lett. 100, 013905 (2008).

[6] Z. Wang, Y. D. Chong, J. D. Joannopoulos, and M. Soljačić, Observation of Unidirectional Backscattering-Immune Topological Electromagnetic States, Nature (London) 461, 772 (2009).

[7] W.-J. Chen, S.-J. Jiang, X.-D. Chen, J.-W. Dong, and C. T. Chan, Experimental Realization of Photonic Topological Insulator in a Uniaxial Metacrystal Waveguide, arXiv: 1401.0367.

[8] M. C. Rechtsman, J. M. Zeuner, Y. Plotnik, Y. Lumer, D. Podolsky, F. Dreisow, S. Nolte, M. Segev, and A. Szameit, Photonic Floquet Topological Insulators, Nature (London) 496, 196 (2013).

[9] M. Hafezi, E. A. Demler, M. D. Lukin, and J. M. Taylor, Robust Optical Delay Lines with Topological Protection, Nat. Phys. 7, 907 (2011).

[10] M. Hafezi, S. Mittal, J. Fan, A. Migdall, and J. M. Taylor, Imaging Topological Edge States in Silicon Photonics, Nat. Photonics 7, 1001 (2013).

[11] K. Fang, Z. Yu, and S. Fan, Realizing Effective Magnetic Field for Photons by Controlling the Phase of Dynamic Modulation, Nat. Photonics 6, 782 (2012).

[12] J. Koch, A. A. Houck, K. L. Hur, and S. M. Girvin, TimeReversal-Symmetry Breaking in Circuit-QED-Based Photon Lattices, Phys. Rev. A 82, 043811 (2010).

[13] A. Petrescu, A. A. Houck, and K. Le Hur, Anomalous Hall Effects of Light and Chiral Edge Modes on the Kagomé Lattice, Phys. Rev. A 86, 053804 (2012).

[14] A. B. Khanikaev, S. H. Mousavi, W.-K. Tse, M. Kargarian, A. H. MacDonald, and G. Shvets, Photonic Topological Insulators, Nat. Mater. 12, 233 (2013).

[15] Y. Lumer, Y. Plotnik, M. C. Rechtsman, and M. Segev, Self-Localized States in Photonic Topological Insulators, Phys. Rev. Lett. 111, 243905 (2013).

[16] K. v. Klitzing, G. Dorda, and M. Pepper, New Method for High-Accuracy Determination of the Fine-Structure Constant Based on Quantized Hall Resistance, Phys. Rev. Lett. 45, 494 (1980).

[17] D. J. Thouless, M. Kohmoto, M. P. Nightingale, and M. den Nijs, Quantized Hall Conductance in a Two-Dimensional Periodic Potential, Phys. Rev. Lett. 49, 405 (1982).

[18] R. B. Laughlin, Quantized Hall Conductivity in Two Dimensions, Phys. Rev. B 23, 5632 (1981).

[19] B. I. Halperin, Quantized Hall Conductance, CurrentCarrying Edge States, and the Existence of Extended States in a Two-Dimensional Disordered Potential, Phys. Rev. B 25, 2185 (1982).

[20] P. W. Brouwer, Scattering Approach to Parametric Pumping, Phys. Rev. B 58, R10135 (1998).

[21] D. Meidan, T. Micklitz, and P. W. Brouwer, Topological Classification of Adiabatic Processes, Phys. Rev. B 84, 195410 (2011).

[22] I. C. Fulga, F. Hassler, and A. R. Akhmerov, Scattering Theory of Topological Insulators and Superconductors, Phys. Rev. B 85, 165409 (2012).

[23] M. Pasek and Y. D. Chong, Network Models of Photonic Floquet Topological Insulators, Phys. Rev. B 89, 075113 (2014).
[24] Y. E. Kraus, Y. Lahini, Z. Ringel, M. Verbin, and O. Zilberberg, Topological States and Adiabatic Pumping in Quasicrystals, Phys. Rev. Lett. 109, 106402 (2012).

[25] M. Verbin, O. Zilberberg, Y. Lahini, Y. E. Kraus, and Y. Silberberg, Topological Pumping over a Photonic Fibonacci Quasicrystal, arXiv:1403.7124.

[26] M. Hafezi, Measuring Topological Invariants in Photonic Systems, Phys. Rev. Lett. 112, 210405 (2014).

[27] T. Oka and H. Aoki, Photovoltaic Hall Effect in Graphene, Phys. Rev. B 79, 081406 (2009).

[28] J. I. Inoue and A. Tanaka, Photoinduced Transition between Conventional and Topological Insulators in Two-Dimensional Electronic Systems, Phys. Rev. Lett. 105, 017401 (2010).

[29] T. Kitagawa, M. S. Rudner, E. Berg, and E. Demler, Exploring Topological Phases with Quantum Walks, Phys. Rev. A 82, 033429 (2010)

[30] T. Kitagawa, E. Berg, M. Rudner, and E. Demler, Topological Characterization of Periodically Driven Quantum Systems, Phys. Rev. B 82, 235114 (2010).

[31] N. H. Lindner, G. Refael, and V. Galitski, Floquet Topological Insulator in Semiconductor Quantum Wells, Nat. Phys. 7, 490 (2011).

[32] Z. Gu, H. A. Fertig, D. P. Arovas, and A. Auerbach, Floquet Spectrum and Transport through an Irradiated Graphene Ribbon, Phys. Rev. Lett. 107, 216601 (2011).

[33] M. S. Rudner, N. H. Lindner, E. Berg, and M. Levin, Anomalous Edge States and the Bulk-Edge Correspondence for Periodically Driven Two-Dimensional Systems, Phys. Rev. X 3, 031005 (2013).

[34] J. T. Chalker and P. D. Coddington, Percolation, Quantum Tunnelling and the Integer Hall Effect, J. Phys. C 21, 2665 (1988).

[35] B. Kramer, T. Ohtsuki, and S. Kettemann, Random Network Models and Quantum Phase Transitions in Two Dimensions, Phys. Rep. 417, 211 (2005).

[36] S. Ryu, C. Mudry, H. Obuse, and A. Furusaki, The $\mathbb{Z}_{2}$ Network Model for the Quantum Spin Hall Effect: Two-Dimensional Dirac Fermions, Topological Quantum Numbers and Corner Multifractality, New J. Phys. 12, 065005 (2010).

[37] G. Q. Liang and Y. D. Chong, Optical Resonator Analog of a Two-Dimensional Topological Insulator, Phys. Rev. Lett. 110, 203904 (2013).

[38] G. Q. Liang and Y. D. Chong, Optical Resonator Analog of a Photonic Topological Insulator: A Finite-Difference Time-Domain Study, Int. J. Mod. Phys. B 28, 1441007 (2014).

[39] T. Ozawa and I. Carusotto, Anomalous and Quantum Hall Effects in Lossy Photonic Lattices, Phys. Rev. Lett. 112, 133902 (2014).

[40] C.-E. Bardyn, S. D. Huber, and O. Zilberberg, Measuring topological invariants in small photonic lattices, New J. Phys. 16, 123013 (2014).

[41] J. Zeuner, M. C. Rechtsman, Y. Plotnik, Y. Lumer, M. S. Rudner, M. Segev, and A. Szameit, Probing Topological Invariants in the Bulk of a Non-Hermitian Optical System, arXiv:1408.2191.

[42] See Supplemental Material at http://link.aps.org/ supplemental/10.1103/PhysRevX.5.011012 for details regarding the simulations of the microwave network. 